$\Rightarrow$ IMMUNE REGULATION

\section{IL-10 targets macrophage metabolism}

Although macrophages are known to be the main target cells of the anti-inflammatory cytokine interleukin-10 (IL-10), little is known about the mechanisms by which IL-10 mediates its effects. Medzhitov and colleagues provide new evidence that IL-10 regulates the metabolic switch to glycolysis that occurs in macrophages that are activated with lipopolysaccharide (LPS) and prevents the accumulation of dysfunctional mitochondria.

After LPS stimulation, $I l 10^{-/-}$ bone marrow-derived macrophages (BMDMs) had higher levels of glycolysis and lower levels of oxidative phosphorylation compared with wild-type BMDMs, as measured by a higher extracellular acidification rate (ECAR) and a lower oxygen consumption rate (OCR), respectively. In wild-type BMDMs, IL-10 inhibited glycolysis by downregulating the translocation of glucose transporter 1 (GLUT1) from intracellular vesicles to the cell surface. Furthermore, IL-10 inhibited the expression of glycolytic pathway genes.
Il10-/- BMDMs had a lower maximal respiratory capacity than wild-type cells after LPS stimulation, which indicates that lack of IL-10 could result in reduced mitochondrial fitness and hence a reduced OCR. The authors therefore investigated whether the altered metabolic profile of $I l 10^{-/-}$BMDMs also results from abnormal mitochondrial function. Il10-/- BMDMs had an increased mitochondrial mass after LPS stimulation compared with wild-type BMDMs due to the accumulation of dysfunctional mitochondria with loss of the mitochondrial membrane potential. This was associated with increased production of mitochondrial reactive oxygen species (ROS).

Further experiments showed that this accumulation of dysfunctional mitochondria in $\mathrm{Il10}^{-1-} \mathrm{BMDMs}$ was the result of impaired mitophagy (the selective degradation of mitochondria by autophagy). Il10-/- BMDMs had significantly lower levels of autophagy than control cells after LPS stimulation. BMDMs lacking autophagy protein 5 (ATG5) had a decreased OCR, similar to $1 l 10^{-/-}$BMDMs, but the

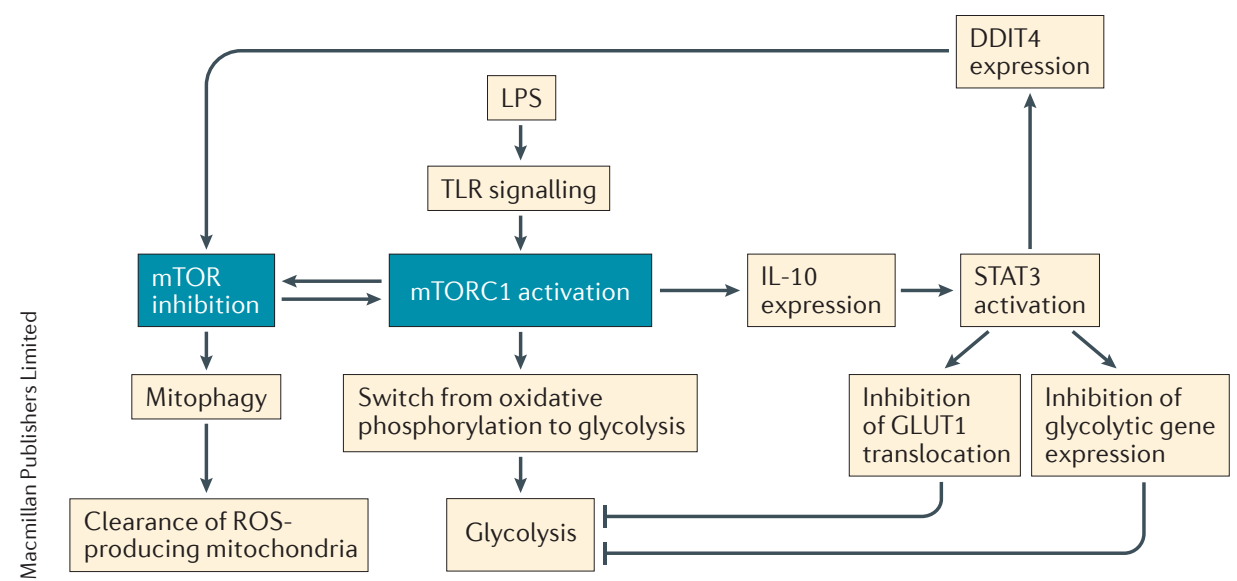

wild-type metabolic phenotype could not be restored in ATG5deficient BMDMs by the addition of exogenous IL-10. Thus, the effect of IL-10 on mitochondrial function is highly dependent on autophagy.

Activation of the mechanistic target of rapamycin (mTOR) complex 1 (mTORC1) promotes glycolysis and inhibits autophagy, and mTORC1 is thus a key potential target for the mitochondrial effects of IL-10. Il10 ${ }^{-/-}$BMDMs - and BMDMs lacking the transcription factor STAT3 (which is activated downstream of the IL-10 receptor) - had increased and prolonged mTORC1 activation after LPS stimulation compared with wild-type cells. Directly inhibiting mTOR with rapamycin in LPSstimulated $\mathrm{IllO}^{-1-}$ BMDMs resulted in decreased accumulation of dysfunctional mitochondria, decreased ECAR and increased OCR. DDIT4 (a known negative regulator of mTOR) was strongly induced by IL-10 in a STAT3-dependent manner during LPS stimulation, and Ddit4 ${ }^{-/-}$BMDMs had prolonged mTORC1 activation during LPS stimulation, which could not be reduced by exogenous IL-10.

The authors conclude that IL-10 inhibits mTORC1 signalling in a STAT3-DDIT4-dependent manner, which is required for the inhibition of glycolysis and the autophagic clearance of dysfunctional, ROS-producing mitochondria. As mitochondrial ROS can function as an endogenous second signal for inflammasome activation, BMDMs lacking IL-10, STAT3 or DDIT4 had increased inflammasome-mediated caspase 1 cleavage and IL- $1 \beta$ production after LPS stimulation as a result of decreased mitophagy. Therefore, the mTORC1-mediated effects of IL-10 on macrophage metabolism have crucial anti-inflammatory roles. 